\title{
Habitable Worlds Around M Dwarf Stars: The CAPSCam Astrometric Planet Search
}

\author{
Alan P. Boss ${ }^{1}$, Alycia J. Weinberger ${ }^{2}$, Guillem Anglada-Escudé ${ }^{3}$, \\ Ian B. Thompson ${ }^{4}$, and Rafael Brahm ${ }^{5}$ \\ ${ }^{1}$ DTM, Carnegie Institution, \\ 5241 Broad Branch Road, N.W., \\ Washington DC, 20015-1305, United States \\ email: boss@dtm.ciw.edu \\ ${ }^{2}$ DTM, Carnegie Institution, \\ 5241 Broad Branch Road, N.W., \\ Washington DC, 20015-1305, United States \\ email: weinberger@dtm.ciw.edu \\ ${ }^{3}$ The Carnegie Observatories, \\ 813 Santa Barbara Street, \\ Pasadena CA, 91101, United States \\ email: ian@obs.carnegiescience.edu \\ ${ }^{4}$ Institut für Astrophysik, Universität Göttingen, \\ Friedrich-Hund-Platz 1, \\ 37077 Göttingen, Germany \\ email: guillem.anglada@gmail.com \\ ${ }^{5}$ Departamento de Astronomía y Astrofísica, Pontificia Universidad Católica de Chile, \\ Av. Vicuña Mackenna 4860, \\ 782-0436 Macul, Santiago, Chile \\ email: rabrahm@uc.cl
}

\begin{abstract}
M}$ dwarf stars are attractive targets in the search for habitable worlds as a result of their relative abundance and proximity, making them likely targets for future direct detection efforts. Hot super-Earths as well as gas giants have already been detected around a number of early M dwarfs, and the former appear to be the high-mass end of the population of rocky, terrestrial exoplanets. The Carnegie Astrometric Planet Search (CAPS) program has been underway since March 2007, searching 100 nearby late M, L, and T dwarfs for gas giant planets on orbits wide enough for habitable worlds to orbit interior to them. The CAPSCam-N camera on the 2.5-m du Pont telescope at the Las Campanas Observatory has demonstrated the ability to detect planets as low in mass as Saturn orbiting at several AU around late M dwarfs within 15 pc. Over the next decade, the CAPS program will provide new constraints on the planetary census around late $\mathrm{M}$ dwarf stars, and hence on the suitability of these nearby planetary systems for supporting life.
\end{abstract}

Keywords. astrometry, planetary systems, planetary systems: formation, stars: low-mass, brown dwarfs

\section{Introduction}

A number of the first hot and warm super-Earth exoplanets discovered by Doppler spectroscopy have been found to have at least one longer period gas giant sibling planet, with several having two (e.g., Gl 876, HD 69830) or even three such siblings (e.g., Mu Ara, $55 \mathrm{Cnc}$ ). The star HD 181433 has an inner 7.5 Earth-mass planet and two outer Jupitermass planets, while HD 47186 has an inner 22 Earth-mass planet and an outer Saturnmass planet (Bouchy et al. 2009). The fact that these super-Earths orbit interior to their 
gas and ice giant companions implies that they formed interior to these companions, as it would be difficult to imagine how they could have formed farther out and subsequently managed to migrate in and around their more massive brethren, thereby re-ordering their radial distributions. Based on the analogous orbital configuration of our Solar System, with inner terrestrial planets, intermediate gas giants, and outer ice giants, such hot and warm super-Earths are likely to be the tip of the spectrum of rocky planets, rather than ice giants that migrated inward and melted. Doppler surveys were the first to estimate that about $\sim 30 \%$ of solar-type stars have super-Earths (Mayor et al. 2009), a result that has been more than confirmed by the ongoing Kepler Mission transit photometry survey (Borucki et al. 2011). Microlensing surveys estimate that $\sim 17 \%$ of late low-mass stars have a gas giant planet, and an even larger fraction ( $\sim 52 \%$ to $62 \%)$ have a superEarth (Cassan et al. 2012). These results combine to suggest that rocky super-Earths are commonplace around low-mass stars, from M dwarfs to solar-like $\mathrm{G}$ dwarfs.

Most nearby stars are low mass $\mathrm{M}$ dwarfs, not $\mathrm{G}$ dwarfs, making nearby $\mathrm{M}$ dwarfs attractive candidates for future space telescopes capable of direct imaging of exoplanets, e.g., by optical coronagraphy. While early $\mathrm{M}$ dwarfs are included on several current optical Doppler survey lists, late $\mathrm{M}$ dwarfs are not, as such red dwarfs are too faint at optical wavelengths. While several efforts are underway to develop instrumentation that will permit high precision Doppler spectroscopy at near-infrared wavelengths, at the moment the only means for discovering planets around nearby late $\mathrm{M}$ dwarfs by a means other than direct detection appears to be ground-based astrometry. Here we describe the current status of one such effort.

\section{CAPSCam Instrument}

In 2002 George D. Gatewood and APB began a high-risk effort to use the $2.5 \mathrm{~m}$ du Pont telescope at Carnegie's Las Campanas Observatory in Chile to undertake a longterm astrometric planet search. While the initial plan was to employ one of Gatewood's Multichannel Astrometric Photometers (Gatewood et al. 1980) on the du Pont, IBB suggested that it would be best to start fresh with an astrometric camera designed specifically for the du Pont. The award of an NSF Advanced Technology and Instrumentation Program grant in 2004, coupled with Carnegie Institution funds, allowed us to develop and build the Carnegie Astrometric Planet Search Camera, CAPSCam, for use on the du Pont (Boss et al. 2009).

The heart of CAPSCam is a HAWAII-2RG array, where HAWAII stands for HgCdTe Astronomical Wide Area Infrared Imaging, and 2RG stands for 2048 x 2048, Reference columns and rows for bias and gain, and Guide window. The CAPSCam array is a HyViSI hybrid array with Si PIN photodiodes. The user-defined guide window (GW) allows for rapid read-out of the target star image, which is essential given that the target stars are close and bright while the background reference stars are distant and faint. Even still, targets earlier than $\sim$ M3.5 tend to be too bright for CAPSCam, even with the shortest possible GW exposure time of $0.2 \mathrm{~s}$. A typical observation for a planet search target consists of four fifteen-minute-long series of images, taken in a 2" x 2" dither pattern, with a total of $\sim 40$ minutes on-sky during the hour-long epoch. The coatings on the astrometric-quality filter window combined with the HyViSI array sensitivity result in an astrometric camera that is optimized for red dwarf target stars, yielding $\sim 70 \%$ quantum efficiency in the pass band of $810 \mathrm{~nm}$ to $910 \mathrm{~nm}$. Further details about CAPSCam may be found in Boss et al. (2009). 


\section{CAPSCam Planet Search Status}

While the characterization of possible target stars for CAPSCam began in earnest on the du Pont in 2003 using the existing Tek5 camera, the CAPSCam era began with the commissioning run in March 2007. CAPSCam has been in operation on the du Pont ever since then, with $\sim 30$ nights allocated per year for the $\sim 100$ target stars on the exoplanet search list. These target stars include $\sim 66 \mathrm{M}$ dwarfs with spectral types between M3.5 and M7, $~ 10$ M8-M9 dwarfs, and $\sim 21 \mathrm{~L}$ and $\sim 3 \mathrm{~T}$ brown dwarfs. Over a hundred other possible target stars have been examined but dropped as a result of being too bright, in fields that are either too crowded or too sparse, or for being too distant: only targets with CAPSCam-derived kinematic parallax distances of $20 \mathrm{pc}$ or less are retained for the long-term exoplanet search. These low mass target stars offer the distinct advantage that the amplitude of the astrometric wobble is inversely proportional to the stellar mass: a $0.1 M_{\odot}$ star will have a wobble that is a factor of 10 times larger than that of a solar-mass star, all other things (i.e., planetary mass and star-planet separation) being equal.

In spite of the fact that astrometric planet searches have been underway for over half a century, to date no planet has been discovered by astrometry in orbit around a single star, though several Doppler-discovered planets have been confirmed by Hubble Space Telescope Fine Guidance Sensor astrometry. Several substellar-mass candidate companions to bright binary stars have been found using differential astrometry by Muterspaugh et al. (2011), but the uncertainty of which star in each binary hosts the candidate,

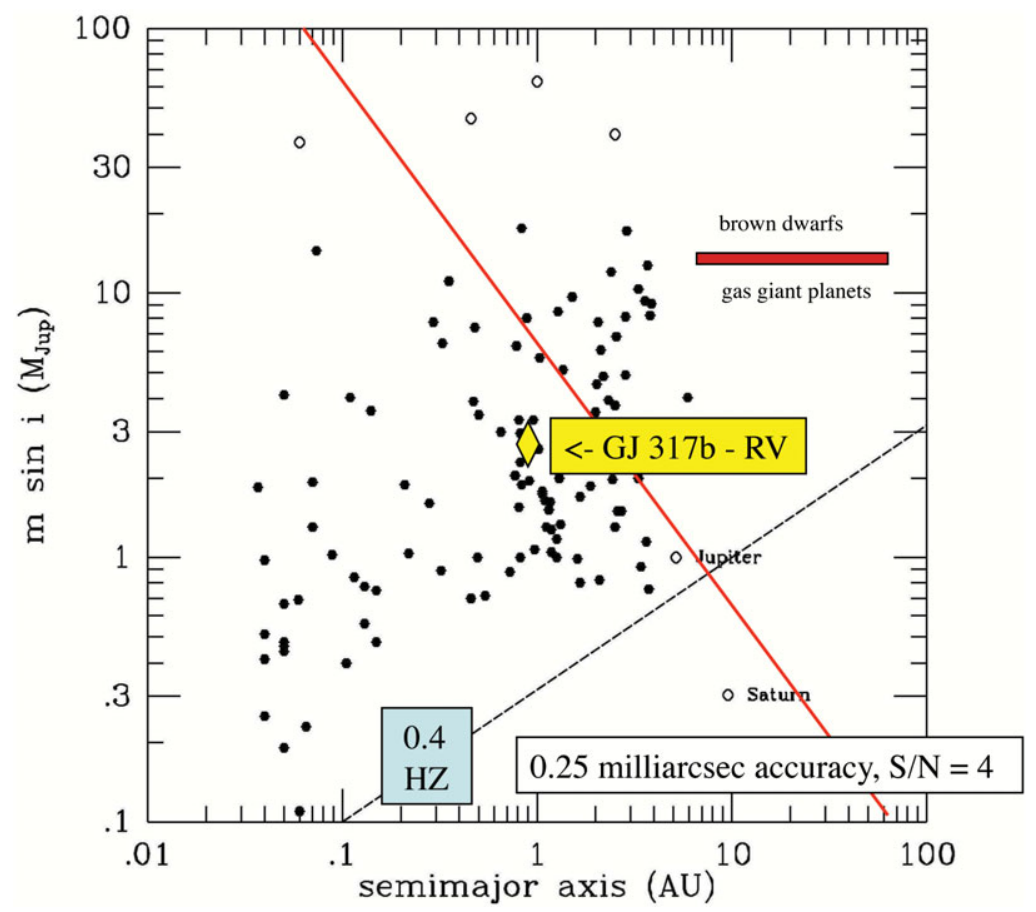

Figure 1. Discovery space plot for Doppler-discovered exoplanets, showing the minimum planet mass in Jupiter mass units as a function of the orbital semimajor axis in AU. The CAPSCam astrometric detection limit (red line) is shown for a $0.4 M_{\odot}$ target star at a distance of $15 \mathrm{pc}$, assuming astrometric accuracy of 0.25 mas and a signal to noise ratio of 4 . Also shown is the approximate radial extent of the habitable zone for a target star with a mass of $0.4 M_{\odot}$, and the CAPSCam-constrained best estimate of the mass of the Doppler-discovered exoplanet GJ317b (Anglada-Escudé et al. 2012a). 
coupled with the absence to date of any independent confirmations, makes the status of these candidates uncertain. The Palomar Testbed Interferometer used for these exacting measurements was closed in 2008, and no other northern hemisphere facility currently exists that is capable of confirming the candidates. The CAPSCam program is motivated in large part by the desire to determine if ground-based astrometry on a relatively modest telescope is indeed capable of discovering extrasolar planets.

Boss et al. (2009) showed that based on the astrometric residuals (after fitting for proper motion and parallax) for a target star (NLTT 48256) that turned out be quite distant $(\sim 55 \mathrm{pc})$, over a two-year period of observations, the long-term astrometric accuracy of CAPSCam appeared to be better than $\sim 0.4$ milliarcseconds (mas), and might be improved to $\sim 0.3$ mas/epoch with further development of the data processing pipeline created by GAE.

Anglada-Escudé et al. (2012a) showed nine CAPSCam target star parallaxes that agree extremely well with previously determined parallaxes displayed on SIMBAD, further demonstrating the CAPSCam astrometric accuracy. In fact, CAPSCam is often employed to determine distances to individual members of nearby clusters of young stars, such as the TW Hydrae association (Weinberger et al. 2013a, 2013b), at distances of 50 to 100 pc, for which four half-hour epochs of observations typically suffices.

Anglada-Escudé et al. (2012a) also presented the results of a CAPSCam search for an astrometric wobble of the $0.4 M_{\odot}$ M3.5 dwarf GJ 317, which was known from Doppler surveys to have a long-period (692 days) gas giant planet with a minimum mass of 1.8 Jupiter masses. GJ 317 was believed to lie at a distance of $\sim 9$ pc, close enough that CAPSCam might be able to detect the astrometric wobble. However, 18 epochs of CAPSCam astrometry spread out over 2.5 years determined that the true distance to GJ 317 is $\sim 15 \mathrm{pc}$, far enough away to significantly reduce the expected astrometric wobble if the orbit was nearly edge-on, i.e., the minimum Doppler mass was close to the true planet mass. CAPSCam did not detect a signal and hence was only able to place a firm upper bound on the mass of the GJ 317b planet of $\sim 3.6$ Jupiter masses, with a most likely mass of $\sim 2.5$ Jupiter masses. Fig. 1 shows the CAPSCam mass constraint for GJ $317 \mathrm{~b}$, and illustrates that there is sufficient interior orbital room for an inner habitable Earth-mass planet to exist. This study also showed that the epoch-to-epoch precision of CAPSCam is 0.9 mas (an average of the precision in RA and Dec), so that with 18 epochs of data, the CAPSCam accuracy should be $\sim 0.2$ mas. For fainter stars where the GW is not needed, we expect this accuracy to be even better, as the GW introduces a small but non-negligible error in the Dec direction.

CAPSCam has been operational for $\sim 5.5$ years, during which $\sim 800$ epochs of observations have been taken on the $\sim 100$ targets on the currently active list. Another $\sim 200$ epochs have been taken on targets that have since been dropped from the planet search list, but for which kinematic parallaxes have been determined and will be published. Assuming that it takes at least about nine epochs to determine the parallax, proper motion in RA and in Dec, and to search for residuals that might be indicative of the presence of planetary or sub-stellar mass companions (i.e., six orbital parameters), the CAPSCam search is just now entering the most exciting phase of this decade-old effort. A number of CAPSCam targets are beginning to show evidence of wobbles that might be caused by unknown companions. Given that astrometry favors the detection of long-period planets, we are concentrating on the targets that show possible long-term wobbles. One of our most promising targets to date is an L5 brown dwarf at a distance of $12 \mathrm{pc}$, which appears to show a wobble with a period of 789 days with a false alarm probability (FAP) of $\sim 3 \%$. Because only 10 epochs of observations have been made to date, we hesitate to call this a "candidate", even though the FAP is relatively small. Doppler confirmation 


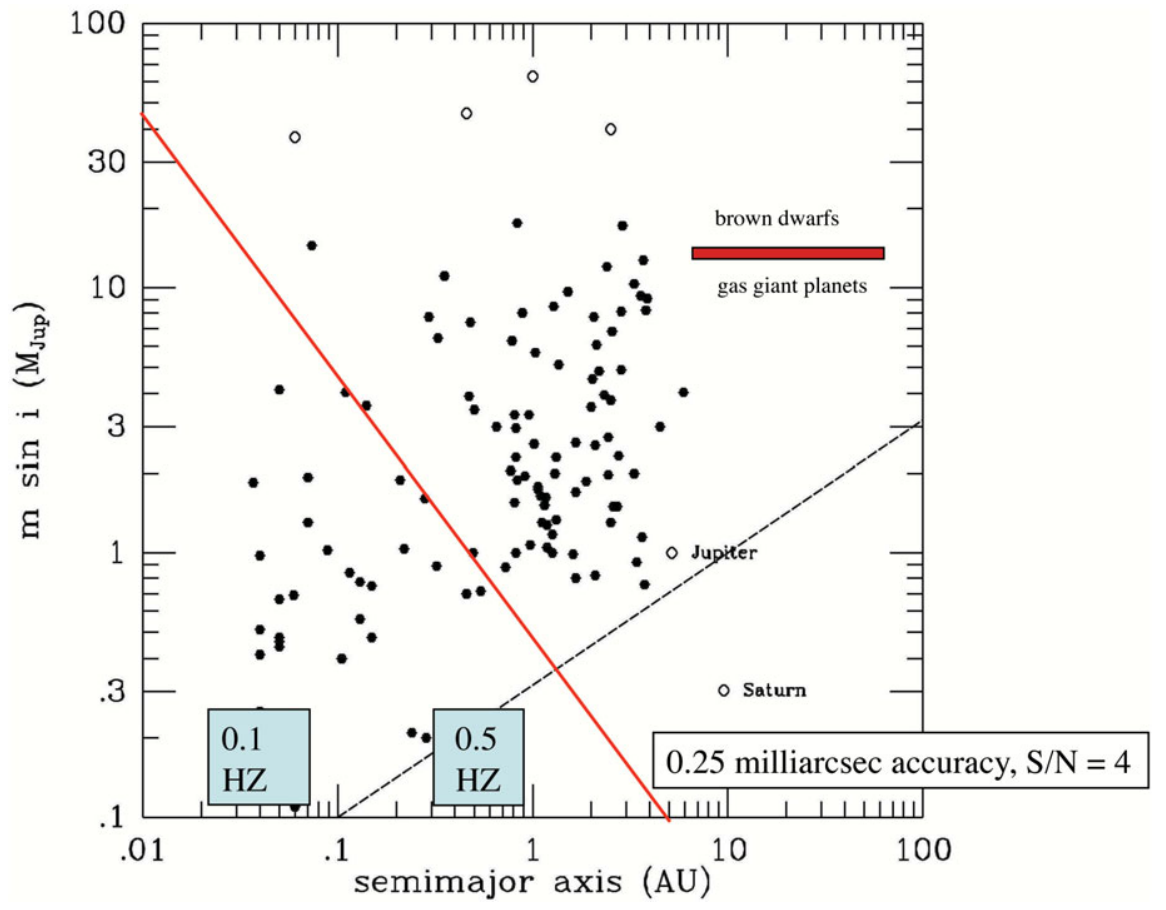

Figure 2. CAPSCam astrometric detection limit (red line) for a $0.1 M_{\odot}$ target star at a distance of $5 \mathrm{pc}$, assuming astrometric accuracy of 0.25 mas and a signal to noise ratio of 4 . Also shown are the approximate radial extents of the habitable zone for target stars with masses of 0.1 and $0.5 M_{\odot}$.

or refutation of the putative astrometric wobble is made difficult by the faintness of this L5 brown dwarf, with I 18, too faint for either the MIKE or PFS spectrographs used on Carnegie's Magellan II Clay telescope at Las Campanas. If the astrometric wobble is correct, for a primary with a mass of $\sim 50$ Jupiter masses, the mass of the secondary would be $\sim 3$ Jupiter masses, in the planetary mass regime, yet not a true "planet" given that the primary is a brown dwarf, rather than a hydrogen-burning star. Further CAPSCam observations are need to clarify the status of this and of several other possible detections that are beginning to emerge from the data.

\section{Conclusions}

From an astrobiological perspective, as a class of stars, there is no apparent reason why many $\mathrm{M}$ dwarfs should not host habitable worlds (Tarter et al. 2007). In fact, much of the excitement in the Doppler search field these days focuses on the question of the possible first discoveries of habitable super-Earths circling $M$ dwarf stars, e.g., GJ 581, which is an M3 dwarf (Mayor et al. 2009; Vogt et al. 2010), and GJ 667, which is an M1.5 dwarf (Bonfils et al. 2011; Anglada-Escudé et al. 2012b). Most of the closest stars are M dwarfs (e.g., Barnard's Star, located 1.8 pc away, is an M4 dwarf), making them tempting targets for direct imaging studies.

CAPSCam has demonstrated (Fig. 2) that it can detect long-period gas giants with masses as small as that of Saturn around late $\mathrm{M}$ dwarf stars within $\sim 15 \mathrm{pc}$ (Boss et al. 2009; Anglada-Escudé et al. 2012a). Doppler surveys have shown that such longperiod planets are frequent siblings to shorter-period super-Earths (Lo Curto et al. 2010). 
Habitable Earths thus are expected to exist in many cases interior to the outer gas giants to be found by CAPSCam astrometry.These nearby $M$ dwarfs should then be prime targets for future space telescopes capable of the direct detection and characterization of habitable Earth-like planets.

\section{References}

Anglada-Escudé, G., et al. 2012a, ApJ, 746, 37

Anglada-Escudé, G., et al. 2012b, ApJL, 751, L16

Bonfils, X., et al. 2011, A\& A, 549, A109

Borucki, W., et al. 2011, ApJ, 736, 19

Boss, A. P., et al. 2009, PASP, 121, 1218

Bouchy, F., et al. 2009, A\&A A, 496, 527

Cassan, A., et al. 2012, Nature, 481, 167

Gatewood, G. D., et al. 1980, Icarus, 41, 205

Lo Curto, G., et al. 2010, $A \& A$, 512, A48

Mayor, M., et al. 2009, A\&\&A, 507, 487

Muterspaugh, M. W., et al. 2011, AJ, 140, 1657

Tarter, J., et al. 2007, Astrobiology, 7, 30

Vogt, S. S., et al. 2010, ApJ, 723, 954

Weinberger, A. J., et al. 2013, ApJ, 762, article id. 118

Weinberger, A. J., et al. 2013, ApJ, 767, article id. 96 